\title{
Diastolic assessment by CZT-SPECT: Could it be the next best thing for the detection of subclinical chemotherapy-induced cardiotoxicity?
}

\author{
Efstathia Andrikopoulou, $\mathrm{MD}^{\mathrm{a}}$ \\ a Brigham and Women's Hospital, Boston \\ Received Jun 13, 2019; accepted Jun 13, 2019 \\ doi: $10.1007 / \mathrm{s} 12350-019-01792-y$
}

\section{See related article, pp. 1193-1201}

Advancements in modern medicine have resulted in a continuously growing population of survivors of adult and pediatric malignancies. ${ }^{1}$ This is paralleled by an increased recognition of the cardiac damage induced by chemotherapy and radiation therapy, collectively termed "cancer therapeutics-related cardiac dysfunction" (CTRCD). ${ }^{2}$ It is now well established that, left untreated, CTRCD may result in numerous adverse cardiovascular effects, namely, left ventricular (LV) dysfunction, congestive heart failure, and ultimately increased cardiovascular mortality. ${ }^{3}$ Myocardial recovery and improved survival rely on early diagnosis of CTRCD and prompt initiation of medical therapy. ${ }^{3}$ Noninvasive cardiac imaging has been traditionally used to detect CTRCD, namely, 2-D echocardiography (Echo), cardiac magnetic resonance (CMR), and radionuclide imaging (multi-gated acquisition, MUGA, and singlephoton emission computed tomography, SPECT). ${ }^{2,4,5}$ These modalities are mostly geared towards quantifying the left ventricular ejection fraction (LVEF), as an index of the status of LV systolic function. ${ }^{4}$ It is becoming increasingly evident though, that reduction in the LVEF may signify advanced and possibly irreversible cardiac damage. This emphasizes the need for detection of markers of subclinical cardiotoxicity.

Reprint requests: Efstathia Andrikopoulou, MD, Brigham and Women's Hospital, Boston, USA; eandrikopoulou@bwh.harvard.edu J Nucl Cardiol 2020;27:1202-6.

$1071-3581 / \$ 34.00$

Copyright (c) 2019 American Society of Nuclear Cardiology.
To this end, research is currently focusing on imaging parameters that may be of diagnostic and prognostic value in the detection of subclinical CTRCD. Echo remains at the center of interest, with studies examining the usefulness of diastolic indices ( $E$ wave, E:A ratio, isovolumetric relaxation time), ${ }^{6}$ as well as performing stress Echo $^{7,8}$ and Echo combined with assessment of strain. ${ }^{9,10}$ The gold standard for non-invasive imaging assessment of CTRCD is CMR since it not only enables precise measurement of the $\mathrm{LV}$ and right ventricular (RV) volumes and systolic function (LVEF and RVEF), but it also allows for tissue characterization, using late gadolinium enhancement images (LGE) and T2-weighted sequences. ${ }^{2,4}$ In addition, novel CMR techniques have shown promise in detecting subclinical CTRCD, namely, CMR-based strain and use of T1 and T2 mapping for the quantification of myocardial fibrosis and edema, respectively; however, larger studies are needed to establish their value. ${ }^{11,12}$

Radionuclide imaging techniques have long been utilized for the work-up of patients with known or suspected CTRCD. ${ }^{4}$ MUGA can be used as an alternative to Echo and CMR and is known for its accurate and reproducible volumetric $\mathrm{LV}$ and $\mathrm{RV}$ quantification, as well as LVEF and RVEF. ${ }^{2,4,13,14}$ The use of SPECT was also considered as one of the main modalities for the evaluation of CTRCD until the most recent ASCO guidelines. $^{4,15}$ Despite their advantages, neither MUGA nor SPECT have been consistently shown to accurately detect the development of subclinical CTRCD.

\section{DIASTOLIC ASSESSMENT WITH CZT-SPECT TECHNOLOGY}

The introduction of cadmium-zinc-telluride (CZT) SPECT detector technology enabled accelerated, lowdose cardiac radionuclide imaging. ${ }^{16-18}$ In addition to 
detecting perfusion defects, CZT-SPECT detectors allow for more precise and sophisticated evaluation of LV systolic and diastolic function, with studies showing high interstudy reproducibility of CZT-based volumetric assessment $^{18}$ and good correlation of LVEF and regional wall motion when comparing CZT-SPECT to CMR. ${ }^{19}$ In addition, LV filling indices derived from CZT-SPECT, namely, peak filling rate (PFR) and time to PFR were shown to correlate well with echo-derived diastolic markers. ${ }^{20}$ Combined with the improved spatial and energy resolution of CZT cameras, and low radiation exposure, these results highlight the potential of CZTSPECT for the diagnostic and prognostic work-up of a wider range of indications, besides myocardial ischemia.

In this study, Hansen et al. use CZT-SPECT detector cameras to define age- and gender-specific reference values of radionuclide-derived diastolic indices, namely, left and right ventricular emptying and filling rates (LPER, RPER, LPFR, and RPFR) and atrial filling rates (PFRa) in a population of nondiabetics and cardiovascular disease-free patients $(\mathrm{N}=764)$, prior to undergoing chemotherapy. ${ }^{21}$ In addition, the authors demonstrated high reproducibility of these markers, based on a small $(\mathrm{N}=46)$ subset of patients who underwent serial imaging. ${ }^{21}$ The study has two main advantages: first, it adds to the limited body of literature on the feasibility, accuracy, and reproducibility of a relatively new technology, CZT-SPECT, with regards to $\mathrm{LV}$ and $\mathrm{RV}$ diastolic assessment. This is an extremely crucial task whenever novel techniques and equipment are used to ensure the development of a database that will allow detection of abnormalities. In addition, the authors chose to focus on a patient population of growing importance, namely, cardiovascular disease-free and diabetes-free patients, prior to their first exposure to chemotherapy.

The study is well designed and reported, however, there are a few caveats that should be considered. First, the study size is small considering the goal is to set reference values. The size of each one of the age groups ranged from $\mathrm{N}=12$ (men 30-40 years old) to $\mathrm{N}=116$ (men 60-70 years old). The characterization of the patient population from the perspective of diastolic function could have been more comprehensive, both from a demographic and an imaging standpoint. With respect to the demographics of the participants, special attention should be given to the frequency of hypertension and smoking status, both well-described risk factors for the development of diastolic abnormalities. In the current study, a total of 216 participants ( $28 \%$ of total $\mathrm{N}$ ) were hypertensive and $330(43 \%$ of total $\mathrm{N})$ were smokers. The prevalence of LV diastolic dysfunction in patients with hypertension varies widely, from 18 to $84 \%$, mostly due to the differences in the clinical characteristics of the populations studied and the lack of a unifying definition of diastolic dysfunction among different imaging modalities used to detect and grade it. $^{22}$ With respect to smoking, current smoking status, total years of smoking, and estimated pack-years have all been shown to correlate with worse diastolic function. $^{23}$ The frequency of additional risk factors for diastolic dysfunction, such as obesity, chronic kidney disease, atrial fibrillation, and even dietary habits, namely, dietary sodium intake, ${ }^{22}$ was not reported.

It should also be noted that there was no correlation with Echo-derived diastolic markers. Given that Echo is the mainstay for non-invasive evaluation of diastolic function, comparison with Echo-derived parameters would be of the utmost importance to comprehensively define a population for setting reference values for diastolic function. Furthermore, clinical outcomes were not reported. It would have been useful to know the rates of overt cardiotoxicity, as defined by the current guidelines, i.e., reduction in LVEF, strain, and abnormal biomarkers. This would have provided with preliminary results regarding the potential for use of CZT-SPECTderived LV and RV diastolic indices for the detection of subclinical cardiotoxicity. Therefore, the interpretation of the CZT-SPECT-based LV and RV filling parameters as "normative", in the population under study by Hansen et al. should be performed with extreme caution. Rather than stating that these are indeed normative values, the safest conclusion to draw would be that the data demonstrate the feasibility and reproducibility of quantifying parameters of $\mathrm{LV}$ and $\mathrm{RV}$ dynamic filling using the CZT-SPECT technology by taking advantage of its short acquisition times, low radiation exposure, and high-energy and spatial resolution. The results are promising and could pave the pathway for the use of CZT-SPECT in patients with both ischemic and nonischemic pathologies.

\section{FUTURE DIRECTIONS}

"...treatment can be devised that will work for you... signals of disease are apparent well before the disease appears (at which point it may be too late). We simply need to recognize what those signals are.",

\section{Pieter Cullis}

In the current study, Hansen et al. addressed two important concepts: (a) measurement of markers of LV and RV dynamic filling using CZT-SPECT detector gamma cameras, and (b) evaluation of patients without known cardiovascular disease or diabetes prior to their first exposure to chemotherapy. ${ }^{21}$ The results showed 
that development of a database with reference values for the assessment of diastolic function using CZT technology can be done. This could lead to the expansion of the use of CZT-SPECT in numerous indications. First and foremost, in patients with known or suspected ischemic heart disease, the detection of abnormalities in LV dynamic filling may enable identification of patients with non-obstructive atherosclerosis, thereby enhancing their risk stratification. This was shown in a small study of $(\mathrm{N}=195)$ patients who presented with chest pain underwent both stress-rest CZT-SPECT myocardial scintigraphy and either invasive coronary angiography or coronary computed tomography angiography. ${ }^{24}$ The authors found that abnormalities in LV diastolic function on CZT-SPECT imaging not only correlated with the presence of non-obstructive $(<50 \%$ luminal stenosis $)$ coronary artery disease (CAD), but the severity of diastolic dysfunction was also associated with the extent of perfusion defects. These results indicated that LV PFR could potentially be used as an earlier, more sensitive marker of myocardial ischemia. ${ }^{24}$

Assessment of the diastolic function using CZTSPECT cameras may not only offer a more sensitive and sophisticated means of providing diagnosis and prognosis to patients with known or suspected CAD, but it may also prove useful in a wide range of non-ischemic pathologies. One of the most exponentially recognized one involves patients with new diagnosis of malignancy undergoing chemotherapy and radiation treatment, who are at risk for developing CTRCD. As far as imaging is concerned, CTRCD is diagnosed based on the occurrence of systolic dysfunction, as reflected by a drop in the LVEF and impairment in myocardial strain., ${ }^{2,4}$ MUGA used to be the gold standard for quantification of LVEF in these patients; however, the advancement in Echo and CMR techniques has rendered it outdated. ${ }^{5}$ It should be noted, though, that neither Echo nor CMR is without its own limitations. Acquisition of accurate LVEF based on Echo relies on several factors, such as operator experience and patient body habitus, and in most cases, the LVEF is based on a visual, subjective estimate. Assessment of LV systolic function and quantification of LVEF based on CMR are more objective compared to Echo, but CMR has a few important contraindications: it cannot be performed in patients with non-MR conditional cardiac implantable electronic devices, patients allergic to gadolinium contrast, and patients with claustrophobia. In addition, it is avoided in patients with advanced renal disease for fear of nephrogenic systemic fibrosis. ${ }^{25}$

In addition to the constraints mentioned above, the challenge, faced by Cardio-oncologists and Cardiac imagers today, is the timely identification of patients at risk for CTRCD, ideally at a stage prior to an overt impairment in LV systolic function, indicated by a decrease in the LVEF. This is where the use of CZTSPECT may offer a potentially effective alternative for risk stratifying these patients. In ischemic heart disease, it is well known that diastolic abnormalities far precede the onset of systolic dysfunction, both by strain and by evident wall motion abnormalities and reduction in the LVEF ("ischemic cascade"). A similar concept was tested in patients at risk for CTRCD, using Echo; however, the results were inconsistent. As a result, the use of Echo-derived diastolic parameters is not recommended by the current guidelines in the setting of workup for possible CTRCD. ${ }^{2,4,5}$ This apparent clinical gap in timely detection of subclinical CTRCD may be filled by the evaluation of $\mathrm{LV}$ and RV dynamic filling by using CZT-SPECT myocardial scintigraphy. In the 1990s, Cottin et al., in two small studies $(\mathrm{N}=60$ and $\mathrm{N}=33$ patients), showed that radionuclide-derived diastolic parameters (PFR, PER, time to PFR, and time to PER) were reduced in patients receiving anthracycline chemotherapy. ${ }^{26,27}$ More recently, in their study of 202 patients, Klein et al. showed that, following exposure to trastuzumab (with or without anthracyclines), patients who have normal diastolic function at baseline may develop diastolic dysfunction prior to evident drop in their LVEF. ${ }^{28}$ These findings solidify the potential role for the new CZT-SPECT technology in the evaluation of patients at risk for CTRCD by a timelier risk stratification of the subset of patients who would benefit from earlier referral to Cardiology, more intensive monitoring, and more aggressive optimization of their risk factors to avoid development of complications.

The question becomes, what do we need to do to better identify these patients at risk? The obvious answer is to design and carry out larger studies to try to elucidate the role of CZT-SPECT in the work-up of patients both at risk for and with known CTRCD. The first step, though, even before this, would be to do what Hansen et al. attempted, which is, to develop a database with reference values for markers of both LV and RV dynamic filling. This will require a comprehensive characterization of the population studied, which will in turn enable the use of the data in the full spectrum of cardiovascular pathologies. Following this, the focus can shift to each specific clinical entity, such as non-obstructive coronary artery disease, CTRCD, valvular heart disease, congenital heart disease (taking advantage of the RV filling indices), and even hypertrophic, infiltrative, and restrictive cardiomyopathies.

On a final note, it is of critical importance to take advantage of all the tools at our disposal. Multimodality cardiovascular imaging can truly be indispensable in the 
Table 1. Comparison of Echo, CMR, and CZT-SPECT myocardial imaging in the assessment of patients with known or suspected CTRCD

\begin{tabular}{|c|c|c|c|}
\hline Modality & Echo & CMR & CZT-SPECT \\
\hline LV, RV volumes & $\bullet$ & $\bullet \bullet$ & - (Usually underestimated) \\
\hline LVEF, RVEF & $\bullet$ & $\bullet \bullet$ & $\bullet$ \\
\hline LV diastolic function & $\bullet \bullet$ & & $\bullet$ \\
\hline RV diastolic function & & & $\bullet$ \\
\hline Fibrosis, edema & & $\bullet \bullet \bullet$ & \\
\hline Strain assessment & $\bullet \bullet$ & $\bullet \bullet$ & \\
\hline Radiation exposure & & & $\bullet$ \\
\hline Contrast administration & & $\bullet \bullet$ & \\
\hline Additional notes & $\begin{array}{l}\text { Cost-effective } \\
\text { Widely available } \\
\text { Intra- and inter- } \\
\text { observer variability }\end{array}$ & $\begin{array}{l}\text { Gold standard for LV/RV } \\
\text { volumes and EF } \\
\text { Expensive } \\
\text { Not widely available yet } \\
\text { Cannot do in patients with: } \\
\text { claustrophobia } \\
\text { Advanced CKD } \\
\text { Non-MR conditional CIEDs }\end{array}$ & $\begin{array}{l}\text { New technology } \\
\text { Databases with reference } \\
\text { values for LV and RV } \\
\text { dynamic filling not yet available } \\
\text { Radiation exposure } \\
\text { (albeit low dose) } \\
\text { Underestimates LV volumes } \\
\text { compared to Echo and CMR }\end{array}$ \\
\hline
\end{tabular}

$C I E D$, cardiac implantable electronic device; CKD, chronic kidney disease; CMR, cardiac magnetic resonance; CZT-SPECT, cadmium-zinc-telluride single-photon emission computed tomography; Echo, 2D echocardiogram; $L V$, left ventricle; $L V E F$, left ventricular ejection fraction; $R V$, right ventricle; $R V E F$, right ventricular ejection fraction

emerging era of theranostics and precision medicine, and chemotherapy-induced cardiotoxicity is a prime example. Complimentary use of the three modalities (Echo, CMR, and radionuclide imaging) in combination with genetic, metabolic, and biochemical data could enable more personalized risk assessment for each individual patient. CZT-SPECT could prove a valuable addition to our armamentarium, by permitting evaluation of both the LV and RV with respect to their dynamic filling, which has already been shown to precede alterations in systolic function. Table 1 summarizes the pros and cons of each one of the three modalities for the assessment of CTRCD. Future research should focus on the conduction of large-scale, multimodality trials to better define the optimal combination of data that will facilitate more refined and sophisticated risk stratification.

\section{Disclosure}

Efstathia Andrikopoulou does not have any relevant disclosures.

\section{References}

1. Miller KD, Siegel RL, Lin CC, et al. Cancer treatment and survivorship statistics, 2016. CA Cancer J Clin 2016;66:271-89.

2. Zamorano JL, Lancellotti P, Rodriguez Muñoz D, et al. 2016 ESC Position Paper on cancer treatments and cardiovascular toxicity developed under the auspices of the ESC Committee for Practice Guidelines: The Task Force for cancer treatments and cardiovascular toxicity of the European Society of Cardiology (ESC). Eur J Heart Fail 2017;19:9-42.

3. Cardinale D, Colombo A, Lamantia G, et al. Anthracycline-induced cardiomyopathy: clinical relevance and response to pharmacologic therapy. J Am Coll Cardiol 2010;55:213-20.

4. Armenian SH, Lacchetti C, Barac A, et al. Prevention and monitoring of cardiac dysfunction in survivors of adult cancers: American Society of Clinical Oncology Clinical Practice Guideline. J Clin Oncol 2017;35:893-11.

5. Plana JC, Galderisi M, Barac A, et al. Expert consensus for multimodality imaging evaluation of adult patients during and after cancer therapy: a report from the American Society of Echocardiography and the European Association of Cardiovascular Imaging. J Am Soc Echocardiogr 2014;27:91139.

6. Dorup I, Levitt G, Sullivan I, et al. Prospective longitudinal assessment of late anthracycline cardiotoxicity after childhood cancer: the role of diastolic function. Heart 2004;90:12146.

7. Jarfelt M, Kujacic V, Holmgren D, et al. Exercise echocardiography reveals subclinical cardiac dysfunction in young adult 
survivors of childhood acute lymphoblastic leukemia. Pediatr Blood Cancer 2007;49:83540.

8. Bountioukos M, Doorduijn JK, Roelandt JR, et al. Repetitive dobutamine stress echocardiography for the prediction of anthracycline cardiotoxicity. Eur J Echocardiogr 2003;4:300-5.

9. Thavendiranathan P, Poulin F, Lim KD, et al. Use of myocardial strain imaging by echocardiography for the early detection of cardiotoxicity in patients during and after cancer chemotherapy: a systematic review. J Am Coll Cardiol 2014;63:2751-68.

10. Santoro C, Arpino G, Esposito R, et al. 2D and 3D strain for detection of subclinical anthracycline cardiotoxicity in breast cancer patients: a balance with feasibility. Eur Heart J Cardiovasc Imaging 2017;18:930-6.

11. Jordan JH, Vasu S, Morgan TM, et al. Anthracycline-associated T1 mapping characteristics are elevated independent of the presence of cardiovascular comorbidities in cancer survivors. Circ Cardiovasc Imaging 2016;9:1-19.

12. Bogachkov A, Lin K, Freed BH, et al. Myocardial strain analysis with CMR in cardiotoxicity patients using deformation field analysis: comparison to healthy volunteers and heart transplant patients. J Cardiovasc Magn Reson 2016;27:W30.

13. Urena P, Lamas G, Mitchell G, et al. Ejection fraction by radionuclide ventriculography and contrast left ventriculogram. A tale of two techniques. SAVE Investigators. Survival and ventricular enlargement. J Am Coll Cardiol 1999;33:180-5.

14. Mogelvang J, Stokholm KH, Saunamaki K, et al. Assessment of left ventricular volumes by magnetic resonance in comparison with radionuclide angiography, contrast angiography and echocardiography. Eur Heart J 1992;13:1677-83.

15. Mitani I, Jain D, Joska TM, Burtness B, et al. Doxorubicin cardiotoxicity: prevention of congestive heart failure with serial cardiac function monitoring with equilibrium radionuclide angiocardiography in the current era. J Nucl Cardiol 2003;10:132-9.

16. Garcia EV, Faber TL, Esteves FP. Cardiac dedicated ultrafast SPECT cameras: new designs and clinical implications. J Nucl Med 2011;52:210-7.

17. Esteves FP, Raggi P, Folks RD, et al. Novel solid-state-detector dedicated cardiac camera for fast myocardial perfusion imaging: multicenter comparison with standard dual detector cameras. J Nucl Cardiol 2009;16:927-34.

18. Jensen MM, Haase C, Zerahn B. Interstudy repeatability of left and right ventricular volume estimations by serial-gated tomographic radionuclide angiographies using a cadmium-zinc- telluride detector gamma camera. Clin Physiol Funct Imaging 2015;35(6):418-24.

19. Cochet H, Bullier E, Gerbaud E, et al. Absolute quantification of left ventricular global and regional function at nuclear MPI using ultrafast CZT SPECT: initial validation versus cardiac MR. J Nucl Med 2013;54:556-63.

20. Gimelli A, Liga R, Pasanisi EM, et al. Evaluation of left ventricular diastolic function with a dedicated cadmium-zinc-telluride cardiac camera: Comparison with Doppler echocardiography. Eur Heart J Cardiovasc Imaging 2014;15:972-9.

21. Hansen NL, Haarmark C, Zerahn B. Ventricular peak emptying and filling rates measured by gated radionuclide tomographic angiography using a cadmium-zinc-telluride SPECT camera in chemotherapy-naïve cancer patients. J Nucl Cardiol 2019. https://d oi.org/10.1007/s12350-019-01756-2.

22. Nadruz W, Shah AM, Solomon SD. Diastolic dysfunction and hypertension. Med Clin N Am 2017;101:7-17.

23. Nadruz W Jr, Claggett B, Gonçalves A, et al. Smoking and cardiac structure and function in the elderly: The ARIC Study (Atherosclerosis Risk in Communities). Circ Cardiovasc Imaging 2016;9:e004950.

24. Gimelli A, Liga R, Pasanisi EM, et al. Myocardial ischemia in the absence of obstructive coronary lesion: The role of post-stress diastolic dysfunction in detecting early coronary atherosclerosis. $\mathbf{J}$ Nucl Cardiol. 2017;24:1542-50.

25. Peak AS, Sheller A. Risk factors for developing gadolinium-induced nephrogenic systemic fibrosis. Ann Pharmacother 2007;41:1481-5.

26. Cottin Y, Touzery C, Coudert B, et al. Impairment of diastolic function during short-term anthracycline chemotherapy. Br Heart J 1995;73:61-4.

27. Cottin Y, Touzery C, Coudert B, et al. Diastolic or systolic left and right ventricular impairment at moderate doses of anthracycline? A 1-year follow-up study of women. Eur J Nucl Med 1996;23:5116.

28. Klein R, Nadouri D, Osler E, et al. Diastolic dysfunction can precede systolic dysfunction on MUGA in cancer patients receiving trastuzumab-based therapy. Nucl Med Commun 2019;40:22-9.

Publisher's Note Springer Nature remains neutral with regard to jurisdictional claims in published maps and institutional affiliations. 